\title{
EDDY CURRENT EXAMINATION OF STEAM GENERATOR TUBES FROM PHWR POWER PLANTS USING ROTATING MAGNETIC FIELD TRANSDUCER
}

\author{
Raimond Grimberg ${ }^{1}$, Lalita Udpa ${ }^{2}$, Alina Bruma ${ }^{3}$, \\ Rozina Steigmann ${ }^{1}$, Adriana Savin ${ }^{1}$, Satish S. Udpa $^{3}$ \\ ${ }^{1}$ Nondestructive Testing Department,National Institute of $R \& D$ for Technical Physics, \\ 47 D.Mangeron Blvd, Iasi, 700050, ROMANIA \\ ${ }^{2}$ Department of Electrical and Computer Engineering, College of Engineering \\ Michigan State University, 2120 Engineering Building, \\ East Lansing, MI 48824 - 1226, USA \\ ${ }^{3}$ Faculty of Physics, Al.I Cuza University, 11 Carol I Blvd., Iasi, 700506 ROMANIA
}

\begin{abstract}
This paper present the results obtained at examination of steam generator tubes samples made from Incoloy 800 , using eddy current transducer with rotating magnetic field. The emission part creates a magnetic rotating field which induces eddy currents in the walls of tubes, the reception being made with an array of sensors. The method presents the advantages of a complete inspection of tube's surface at one passing. To increase the precision of discontinuity localization, a super resolution algorithm is used. The results are comparables with those obtained at the inspection with rotating probe, being obtained a good correlation, the speed of control being superior in the case of transducer with rotating magnetic field.
\end{abstract}

\section{INTRODUCTION}

At the end of 2006, in the world, 443 Nuclear Power Plants operated in 31 countries, producing approximate $307 \mathrm{GW}(\mathrm{e})$, representing $16 \%$ from the capacity of worldwide electrical energy production [1].

One of classification criterion for nuclear reactors is represented by the type of moderator. The reactors which use heavy water $\left(\mathrm{D}_{2} \mathrm{O}\right)$ as moderator represent an important category, the most known type being the Pressurized Heavy Water Reactor (PHWR) in CANDU variant. This type of reactor use heavy water so as moderator as well as coolant fluid, in two separate circuits:

- the high pressure and high temperature heat transport system (reactor coolant system) that circulates $\mathrm{D}_{2} \mathrm{O}$ coolant through the fuel channels to remove the heat produced by fission in the fuel;

- cool low pressure moderator system that circulates the $\mathrm{D}_{2} \mathrm{O}$ moderator surrounding the fuel channels through the heat exchangers to remove the generated in moderator.

CANDU reactors can operate on natural uranium fuel $\left(\sim 0.7 \% \mathrm{U}^{235}\right)$, or other fuels with low fissile material content.

The CANDU6 (600 MW(e)) heat transport system arrangement comprises two loops. Each loop circulates pressurized heavy water coolant through 190 of the fuel channels located to one side of the vertical centre - plane of the reactor. Each loop contains 2 pumps, 2 steam 
generators, 2 inlet headers and 2 outlet headers, and connecting piping, in a "figure - of eight"; in this arrangement the pumps and steam generators are connected in series, Figure 1 The steam generators, pumps and headers are located above the reactor to facilitate circulation of the coolant by thermosyphoning.

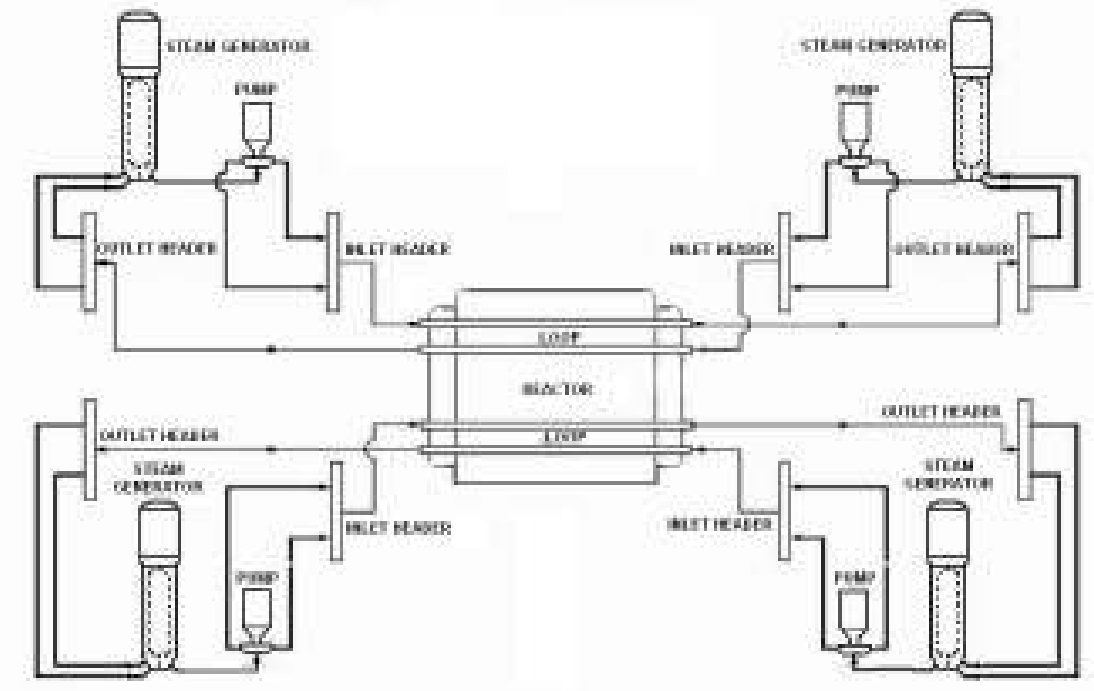

Figure 1.Heat transport system for PHWR nuclear power plant CANDU type

The steam generator is illustrated in Figure 2.

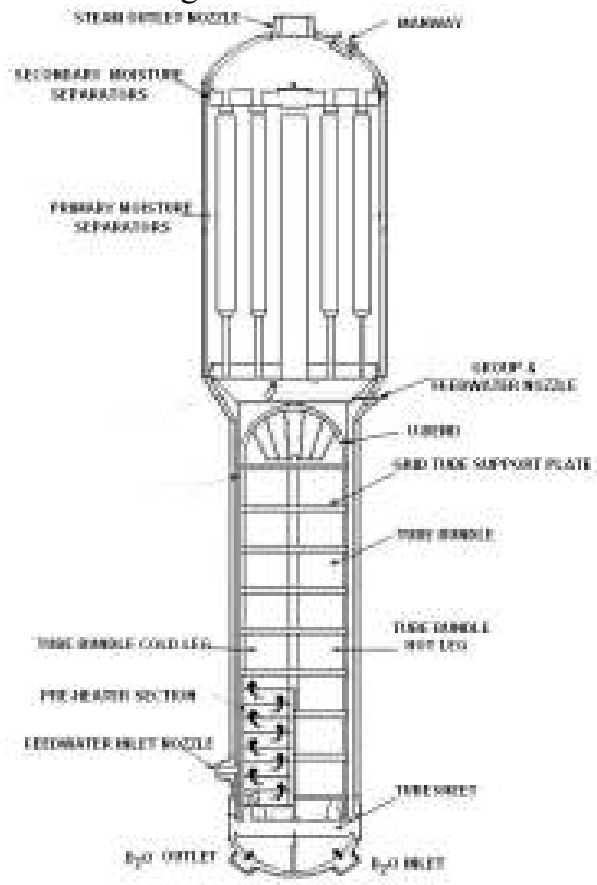

Figure 2. Steam generator 
The steam generator transfer heat from reactor coolant, which flows within the steam generator tubes, to ordinary water surrounding the steam generator tubes on the secondary side to produce steam. CANDU steam generator consists of an inversed vertical U-tube bundle in a cylindrical shell. Steam separating equipment is provided in the steam drum in the upper part of the shell.

The reactor outlet header pressure is $9.9 \mathrm{MPa}$, the total area of transfer heat per steam generator is $3200 \mathrm{~m}^{2}$ and steam pressure is $4.7 \mathrm{MPa}$, at net steam flow rate of $1033 \mathrm{~kg} / \mathrm{sec}$ [2]. The prevention of steam generator tube failure is important in all indirect - cycle nuclear power plants. Thus, careful consideration is given to the secondary side chemistry and to the control of inadvented additions of undesirable chemical species. CANDU secondary side system use All Volatile Treatment and high quality makeup water, exclude alloys containing copper as a major constituent, heave leak tight titanium condensers and optimize operating chemistry to minimize the transfer of corrosion products into steam generator.

The national regulatory bodies in the nuclear field have introduced, as compulsory rule, the in-service inspection (ISI) of steam generator (SG) tubes in the outage period.

Eddy current (EC) testing is the conventional method for IS of SG tubing that is exposed to corrosive, high pressure, and high temperature environment. This non destructive evaluation (NDE) method has advanced significantly over the years in all areas associated with probe design, instrumentation, and computer aided data analyzer. NDE technique is therefore necessary to enhance detection and characterization of difficult flaws.

Bobbin coil probes have long served as the primary NDE tool for initial inspection of SG tubes. Circumferential windings of this probe are concentric with the tube inner surface and render this probe suitable for high speed inspection application. Due to their configuration, bobbin probes are inherently insensitive to narrow circumferential flaws that do not possess axial components [3]. High resolution inspection techniques that utilize multi coils rotating or array configurations are needed to compensate for bobbin coil limitations. Such probes incorporate small coils either as surface riding rotating elements commonly referred to as motorized rotating pancake coil, or multiple element arrays [4],[5]. As a direct consequence of finer spatial resolution, application of such probes requires the use of more efficient signal processing and data screening procedures [6].

We present in this work a new type of EC probe - the transducer with rotating magnetic field and the results obtained at the control of SG tubes.

\section{TRANSDUCER DESCRIPTION}

The transducer construction is presented in Figure 3. The emission part $\mathbf{A}$ consists of three rectangular coils $\mathbf{a}, \mathbf{b}$ and $\mathbf{c}$ with the same turn number, placed $120^{\circ}$ apart. The coils are starconnected and supplied by a three-phase current system. The reception part $\mathbf{B}$ consists of 8 flat coils $1,2, \ldots 6$ placed on the transducer's external surface, connected to a multi - channel eddy current control equipment.

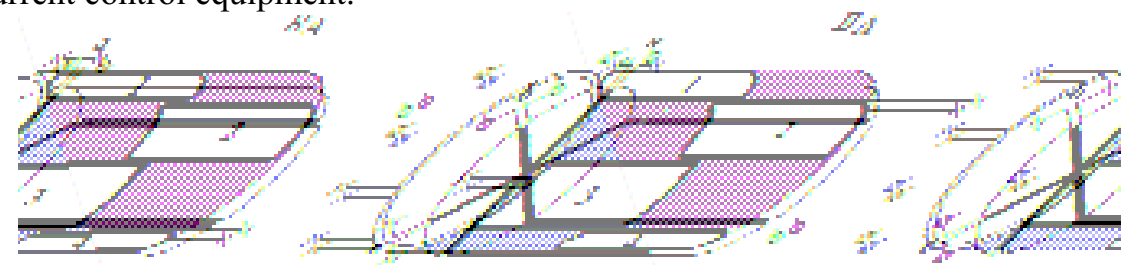

Figure 3 The inside transducer with rotating magnetic field 
The physical realization of the transducer is presented in Figure 4. the theoretical functioning of this transducer was presented in [7].

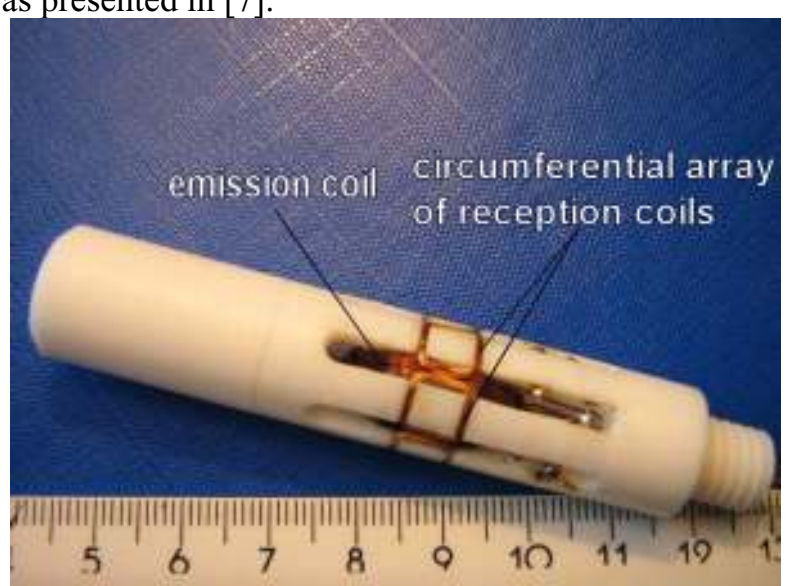

Figure 4. Inner eddy current transducer with rotating magnetic field

\section{SAMPLES AND EXPERIMENTAL SET-UP}

Steam generator tube samples made from Incoloy 800 have the inner diameter $18.2 \mathrm{~mm}$ and wall thickness $1.80 \mathrm{~mm}$. On these samples, artificial discontinuities were practiced using EDM, being presented in Table 1.

Table 1. Dimensions and location of EDM slots

\begin{tabular}{|c|c|c|c|l|}
\hline $\begin{array}{c}\text { Slot } \\
\text { No. }\end{array}$ & $\begin{array}{l}\text { Axial dimension } \\
{[\mathrm{mm}]}\end{array}$ & $\begin{array}{l}\text { Circumferential } \\
\text { dimension }[\mathrm{mm}]\end{array}$ & $\begin{array}{l}\text { Radial dimension } \\
{[\mathrm{mm}]}\end{array}$ & Location \\
\hline 1 & 5 & 0.2 & 0.125 & External surface \\
\hline 2 & 5 & 0.2 & 0.25 & External surface \\
\hline 3 & 5 & 0.2 & 0.5 & External surface \\
\hline 4 & 5 & 0.2 & 0.125 & Internal surface \\
\hline 5 & 5 & 0.2 & 0.25 & Internal surface \\
\hline 6 & 5 & 0.2 & 0.5 & Internal surface \\
\hline 7 & 0.2 & 5 & 0.125 & External surface \\
\hline 8 & 0.2 & 5 & 0.25 & External surface \\
\hline 9 & 0.2 & 5 & 0.5 & External surface \\
\hline 10 & 0.2 & 5 & 0.125 & Internal surface \\
\hline 11 & 0.2 & 5 & 0.25 & Internal surface \\
\hline 12 & 0.2 & 5 & 0.5 & Internal surface \\
\hline
\end{tabular}

The artificial discontinuities presented above were distributed on 4 tube samples having $800 \mathrm{~mm}$ length each, similarly with those used in steam generator construction.

On the tube no.1 were practiced the slots \# 1-\# 6, being aligned after the same generatrix of tube. On the tube no. 2 were practiced the slots \# 7-\# 12, all disposed after the same generatrix, too. These samples have served to verify the response provided by the sensors from the reception array. On the other samples, the slots had different positions. The precision of slots execution was $0.01 \mathrm{~mm}$, the distance between them being $30 \mathrm{~mm}$. Beside these tubes, a steam generator mock-up were used, the tubes having different types of discontinuities (Figure 5). 


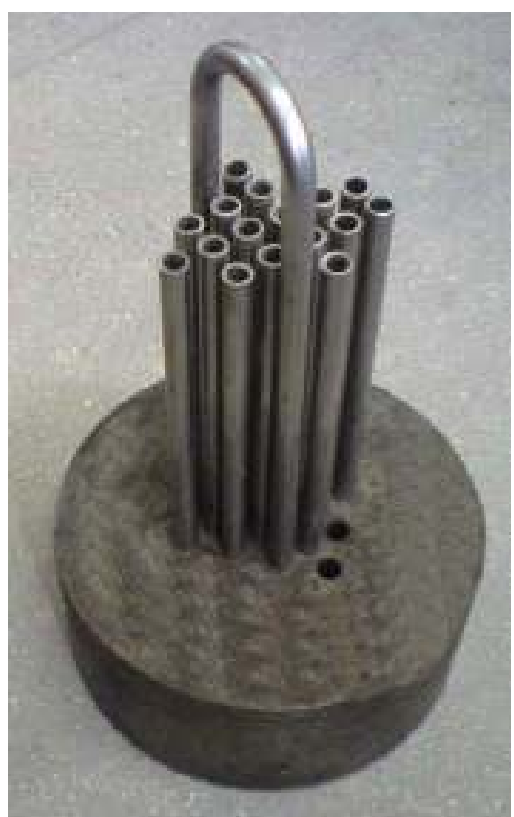

Figure 5. Steam generator mock-up

The principle scheme of the eddy current equipment for steam generator testing using the transducer with rotating magnetic field is presented in Figure 6.

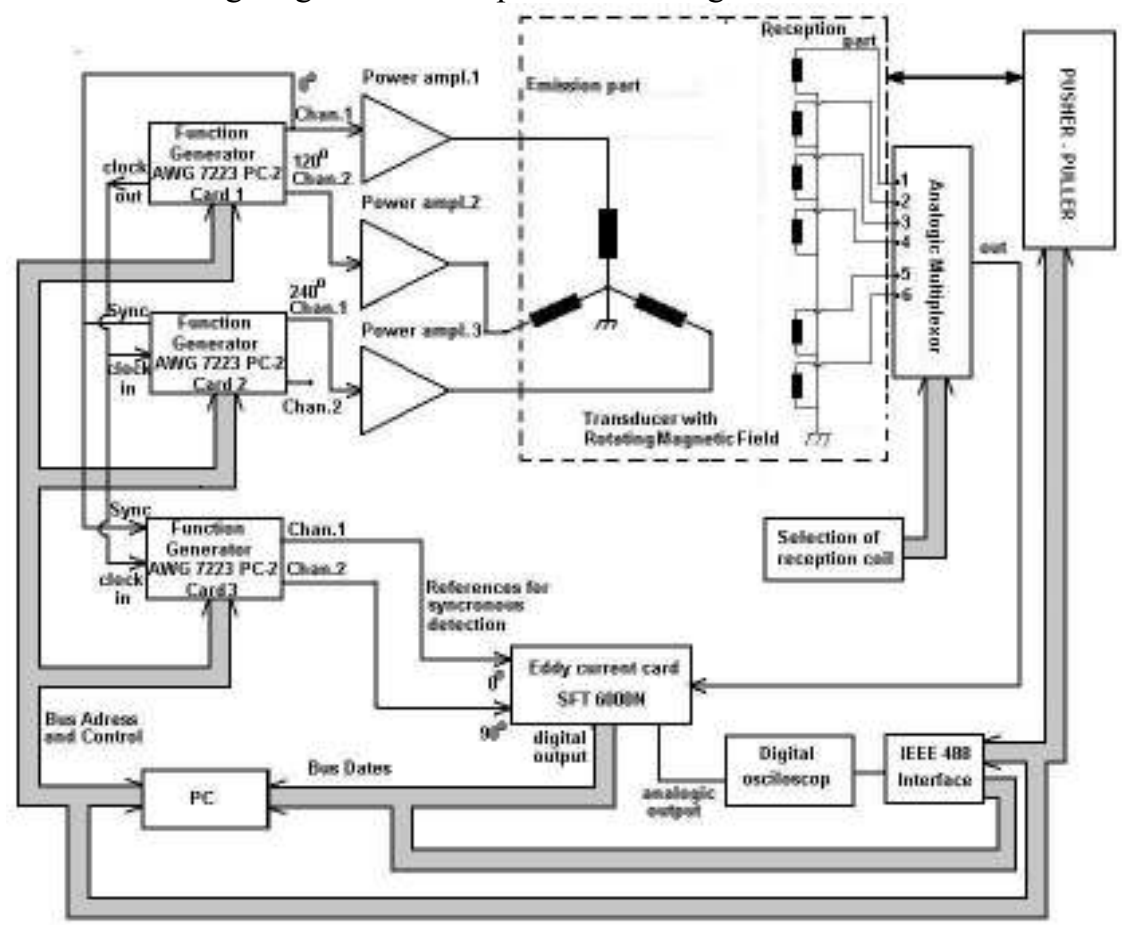

Figure 6.Eddy current equipment lay-out 
Three programmable function generator cards AWG 7223 PC-2 type supply the circuit with the three phase alternative current with equals amplitudes and $2 \pi / 3$ difference of phase between them, as well as the reference for synchronous detection. The signals are amplified and applied to the emission part creating a rotating magnetic field. The cards provide the reference signals for synchronous detectors, included into eddy current SFT $6000 \mathrm{~N}$ card. The 6 receiver coils are consecutively interrogated by means of an analog multiplexer and the signal is applied to the input of an SFT $6000 \mathrm{~N}$ eddy current board. This card contains also pre-amplifiers, filters, direct current amplifiers and a circuit for rotating the phase of the signal. The displacement of the transducer through the tube is made with a push-puller, being commanded by the computer.

\section{SIGNAL PROCESSING SCHEME}

The signal provided by one reception coil from the array is sampled into $\mathrm{n}_{0}$ samples and quantized on 12 bits. The second coil will be interrogated after an interval of time $\mathrm{T}_{0}$ from the moment of the start of first coil interrogation, the operation continuing until the end of the control, on both channel $\mathrm{X}$ and $\mathrm{Y}$ of the equipment.

This situation is presented for one channel, in figure 7.

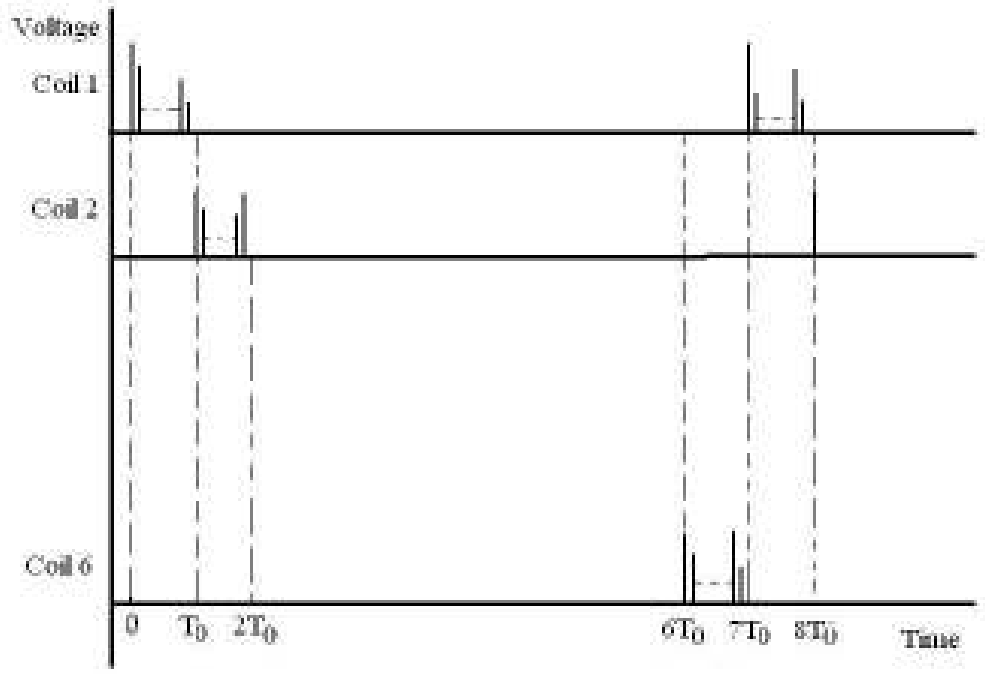

Figure 7. Temporal presentation of data acquisition procedure

Examining the data from the diagram above, it can be observed that each coil is not interrogated during $6 * \mathrm{~T}_{0}$ interval, period in which we don't have information about the state of controlled tube in angular sector of $60^{\circ}$, representing the sensibility region of respective coil.

Only after this time interval, the respective coil will be again interrogated. This disadvantage might be eliminated using a multi-channel equipment but increasing the cost of installation. It results that for the period in which the reception coil is not interrogated, the signal provided by this should be predicted. Using the method of interpolation of the signal starting from the value of the last sample from the period $\mathrm{n}^{*} \mathrm{~T}_{0}$ and the first sample from the immediate period of interrogation $(\mathrm{n}+7) * \mathrm{~T}_{0}$ might be so inefficient, as well as it might introduce big errors. To 
avoid this disadvantage, the effectuation of linear prediction for some time intervals well defined followed by the effectuation of a polynomial interpolation is proposed.

The points where the linear prediction will be made have been chosen as being Chebyshev nodes. In the numerical analysis, the Chebyshev nodes are the roots of the Chebyshev polynomial of the first kind. They are often used as nodes in polynomial interpolation because the resulting interpolation polynomial minimizes the problems of Runge's phenomenon [8]. For a given $\mathrm{n}$, the $\mathrm{n}$ Chebyshev nodes are

$$
x_{i}=\cos \left(\frac{2 i-1}{2 n} \pi\right), i=1,2, \ldots n
$$

All the Chebyshev nodes are contained in the interval $[-1,1]$. To get nodes over an arbitrary interval $[\mathrm{a}, \mathrm{b}]$ a linear transformation can be used

$$
\tilde{x}_{i}=\frac{1}{2}(a+b)+\frac{1}{2}(b-a) \cos \left(\frac{2 i-1}{2 n} \pi\right)
$$

Polynomial interpolation is the interpolation of a given data set by a polynomial. Given some data points, the aim is to find a polynomial which goes exactly through these points. Given a set of $\mathrm{n}+1$ data points $\left(\mathrm{x}_{\mathrm{i}}, \mathrm{y}_{\mathrm{i}}\right)$ where no two $\mathrm{x}_{\mathrm{i}}$ are the same, are is looking for a polynomial $\mathrm{p}$, of degree at most $\mathrm{n}$ with the property

$$
p\left(x_{i}\right)=y_{i}, i=0,1, \ldots, n
$$

The unisolvence theorem states that such polynomial $\mathrm{p}$ exists and is unique.

Linear prediction is a mathematical operation where the future values of a discrete-time signal are obtained as a linear function of previous samples.

The most common representation is

$$
\hat{y}(n)=-\sum_{i=1}^{p} a_{i} y(n-i)
$$

where $\hat{y}(n)$ is the predicted signal value, $\mathrm{y}(\mathrm{n}-\mathrm{i})$ the previous observed values, and $\mathrm{a}_{\mathrm{i}}$ the prediction coefficients. The error generated by this estimation is

$$
e(n)=y(n)-\hat{y}(n)
$$

where $y(n)$ is the thrue signal value.

The most common choice in optimization of predictors' coefficients is the root mean square criterion. In this method we minimize the expected value of the squared error, which yields the equation

$$
\sum_{i=1}^{p} a_{i} R(i-j)=-R(j)
$$

For $1 \leq j \leq p$, where $\mathrm{R}$ is the autocorrelation matrix of signal $\mathrm{y}(\mathrm{n})$, defined as

$$
R(i)=E\{y(n) y(n-i)\}
$$

where $E$ is the expected value, and $R(p+1) x(p+1)$ matrix. The equations (6) and (7) are called Yule-Walker equations or normal equations.

Introducing (4) in (5)

$$
e(n)=y(n)-\hat{y}(n)=y(n)+\sum_{i=1}^{p} a_{i} y(n-i)=\sum_{i=0}^{p} a_{i} y(n-i)
$$

were we usually constrain the parameters $\mathrm{a}_{\mathrm{i}}$ with $\mathrm{a}_{0}=1$, to avoid non trivial solution. In this case the Yule-Walker equation is

$$
R a_{i}=[1,0,0, \ldots 0]^{T}
$$

where the index $i$ ranges from 0 to $p$ and $R$ is $a(p+1) x(p+1)$ matrix. 
For solving (9), we are using the split Levinson recursion [9].

For the case of the transducer with rotating magnetic field, the axial speed for the displacing of the transducer inside the tube was $100 \mathrm{~mm} / \mathrm{sec}$. The sampling rate has been chosen at $100 \mathrm{ksamples} / \mathrm{sec}$. The number of samples for an interrogation period of a reception coil has been set at 100 samples, and the time interval $T_{0}$ between two successive interrogations has been $7 \mathrm{msec}$. The number of Chebyshev nodes has bees selected as $\mathrm{p}=5$.

In this condition the Chebyshev nodes are

$$
x_{i}=3.5 * T_{0}+2.5 * T_{0} * \cos \left(\frac{2 i-1}{10} \pi\right)
$$

For this points a linear prediction for established the values of voltage samples using split Levinson recursion has been made. After this, a polynomial interpolation using polynomial of 5 degree has been made.

\section{EXPERIMENTAL RESULTS}

The results of eddy current examination of the samples 1 and respective 2, using the transducer with rotating magnetic field are presented in Figures 8 and 9. The testing was made at $200 \mathrm{kHz}$, the amplitude of alternative current through the three emission coils being $0.1 \mathrm{~A}$.

The signals provided by the reception coils were amplified with $42 \mathrm{~dB}$ gain, and, after the synchronous detection, were filtered with low pass filter with $20 \mathrm{~Hz}$ cut-off frequency, and then the data were digital scaled to fit in interval $\pm 10 \mathrm{~V}$.

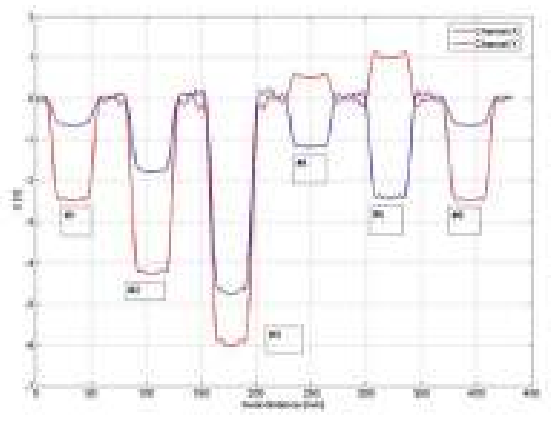

Figure 8 . The data provided by the equipment at the testing of sample no.1 (axial slots with different depths, placed on the external and internal surface of tube. The numbers noted with \# from image, correspond to the slots from Table 1

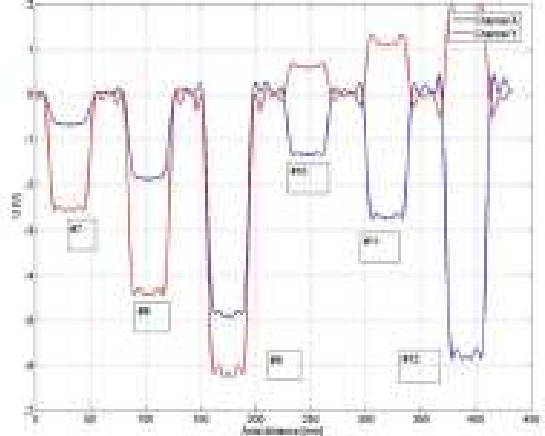

Figure 9 . The data provided by the equipment at the testing of sample no.2 (circumferential slots with different depths, placed on the external and internal surface of tube. The numbers noted with \# from image, correspond to the slots from Table 1.

The examination of the data from the figures above proves the capability of the equipment and the transducer to emphasize axial and circumferential slots with $0.125 \mathrm{~mm}$ depths, representing $10 \%$ from tube's wall thickness, indifferent if they are placed on the internal or on the external surface of the tube. Also, the aspect of data proves the righteous of our processing method based on linear prediction followed by polynomial interpolation.

The data obtained from the examination of samples 3 and for are presented in figures 10 and respective 11 . 

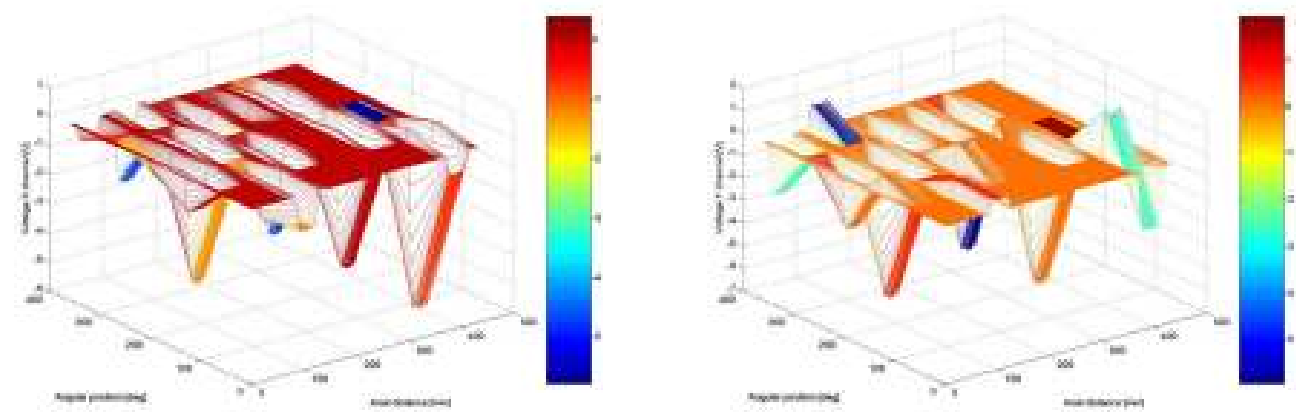

Figure 10. The data provided by the equipment at the testing of sample no.3 :a) channel X; b)channel Y
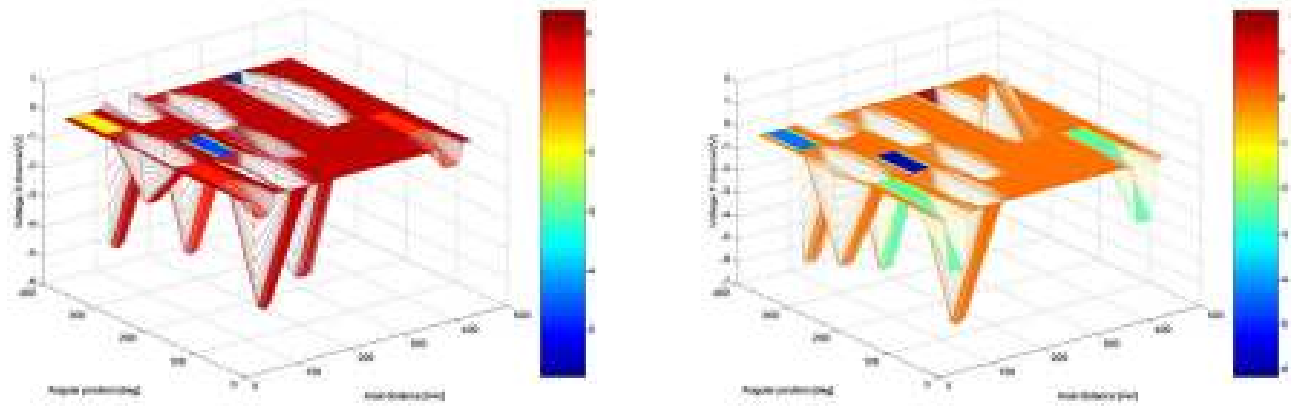

Figure 11. The data provided by the equipment at the testing of sample no.4 :a) channel X; b)channel Y

The examination of the results obtained from the control of tubes samples from steam generator CANDU 6 type, using the inner eddy current transducer with rotating magnetic field, shows that discontinuities with depths of approximate $10 \%$ from tube's wall thickness can be clearly emphasized with a signal to noise ratio better than 5:1. In the same time, the method has the advantage of being rapidly, the axial speed for displacing the transducer inside the tube being comparable with one of eddy current bobbin coil transducer, allowing in the same time the angular localization of the discontinuities with a precision that depends by the number of reception coils.

\section{CONCLUSIONS}

Using the transducer with rotating magnetic field at the examination of tubes from steam generator allows the detection of discontinuities with depths greater or equal with $10 \%$ from tube's wall thickness. The localization of angular position of discontinuities is made in sectors of $60^{\circ}$ angle each, fact possible only in the case of rotating pancake probe. In this case, the transducer has a axial displacing speed lower and contain rotating parts which reduces the reliability 


\section{AKNOWLEDGEMENTS}

This paper is partially supported by Romanian Ministry of Education and Research under Excellence Research Program - Contract no. 6110/2005 CEEX CALIST-Project SINERMAT, and Nucleus Program, Contract no.06-38-01.03

\section{REFERENCE:}

1. Global development of advanced nuclear power plants and related IAEA activities, IAEA, Vienna, 2006.

2. G.Hongqi, R.S.Hart - Moderator, Heat Transport and Steam System, AECL Report, 1992

3. V S Cecco, G Van Drunnen, F L Sharp, 'Manual on eddy current method', Vol 1, AECL Report 7523, Chalk River Nuclear Laboratories, 1981

4. Pressurized Water Reactor Steam Generator Examination Guidelines, Rev.6, Requirements,.EPRI, Palo Alto, CA: 2002.1003138

5. Eddy Current Data Quality Parameters for Inspection of Steam generator Tubes: Vol.4, Array Probes, EPRI, Palo Alto, CA: 2002.1007314

6. S.Bakhtiary, Advanced Eddy Current NDE for Steam Generator Tubing, Report ANL/ET/CP 98667, Aragonne National Laboratory, 1999

7. R. Grimberg, L. Udpa, A. Savin, R. Steigmann, S. Udpa, Inner eddy current transducer with rotating magnetic field. Theoretical model - forward problem, Research in Nondestructive Evaluation, 2005, Springer-Verlag New York, LLC, vol 16, issue 2, pp.79-100

8. Christopher J. Zarowski, An Introduction to Numerical Analysis for Electrical and Computer Engineers John Wiley \& Sons, Inc. 2004

9. N Levinson, "The Wiener RMS error criterion in filter design and prediction," J. Math. Phys., v. 25, 1947., pp. 261-278. 